\title{
Study of a-Si crystallization dependence on power and irradiation time using a $\mathrm{CW}$ green laser
}

\author{
M. Morales*a, D. Munoz-Martin ${ }^{\mathrm{a}}$, Y. Chen ${ }^{\mathrm{a}}$, O. García ${ }^{\mathrm{a}}$, \\ J.J. García-Ballesteros ${ }^{\mathrm{a}}$, J. Cárabe ${ }^{\mathrm{b}}$, J.J. Gandía ${ }^{\mathrm{b}}$, C. Molpeceres ${ }^{\mathrm{a}}$ \\ ${ }^{a}$ Centro Láser UPM, Universidad Politécnica de Madrid, Campus Sur UPM, \\ Ed. La Arboleda, Ctra. de Valencia, km, 7,300, 28031 Madrid, Spain \\ ${ }^{\mathrm{b}}$ CIEMAT, Av. Complutense 40, 28040 Madrid, Spain
}

\begin{abstract}
An advantage of laser crystallization over conventional heating methods is its ability to limit rapid heating and cooling to thin surface layers. Laser energy is used to heat the a-Si thin film to change the microstructure to poly-Si.

Thin film samples of a-Si were irradiated with a CW-green laser source. Laser irradiated spots were produced by using different laser powers and irradiation times.

These parameters are identified as key variables in the crystallization process. The power threshold for crystallization is reduced as the irradiation time is increased. When this threshold is reached the crystalline fraction increases lineally with power for each irradiation time.

The experimental results are analysed with the aid of a numerical thermal model and the presence of two crystallization mechanisms are observed: one due to melting and the other due to solid phase transformation.
\end{abstract}

Keywords: amorphous silicon, polycrystalline silicon, crystallization, annealing, laser, simulation

\section{INTRODUCTION}

Laser assisted crystallization and annealing, as a process to produce polycrystalline silicon (poly-Si) thin films from amorphous-silicon (a-Si), has been a subject of intense research in recent years for a number of applications, including thin-film transistors for active-matrix liquid-crystal displays (AMLCD), sensors and solar cells [1-4].

Thin-film polycrystalline silicon solar cells have long been considered a potential competitor to standard, wafer-based silicon photovoltaics. However ${ }_{2}$ in order to improve the efficiency and lowering cost of these solar cells ${ }_{2}$ intensive researches are done to enhance the quality of polycrystalline silicon films deposited on low cost substrates such as glass.

Laser crystallization of amorphous silicon (a-Si) is regarded as the most efficient method for fabricating polycrystalline silicon on glass [5]. A major advantage of laser crystallization and annealing over conventional heating methods is its ability to limit rapid heating and cooling to thin surface layers. This is mainly controlled by the pulse duration time and the absorption depth of the laser light used in the material. Moreover, large areas can be also crystallized if a laser spot is scanned in overlapping rows onto the sample surface.

Pulsed excimer lasers such as $\mathrm{XeCl}$ and $\mathrm{KrF}$ have been widely used for annealing of a-Si films [6-8] due mainly to the strong absorption of UV light in silicon. However, the traditionally used excimer laser crystallization (ELC) has some disadvantages, mainly the narrow process window of laser energy density and the maximum crystallization depth achievable with this method. In recent works other lasers have been studied, like pulsed Nd:YAG lasers (1064 nm, 532 $\mathrm{nm}$ and $355 \mathrm{~nm}$ ) [9-10], CW green laser [11-15], and CW diode lasers (808 nm) [16-17]. Laser silicon crystallization using these wavelengths and pulse times allows a large variety of grains structure depending on the laser power and the scanning speed, leading to a very wide processing window [18], in opposite to the conventional excimer laser crystallization (ELC) process where the super lateral growth (SLG) regime is very difficult to determine [5].

In laser crystallization processes, the incident energy of the laser pulse is partially absorbed near the surface, heating the sample. The irradiated area increases its temperature even up to melting the a-Si and the heat generated at the surface

Laser-based Micro- and Nanoprocessing VIII, edited by Udo Klotzbach, Kunihiko Washio, Craig B. Arnold, Proc. of SPIE Vol. 8968, 89680U · (C) 2014 SPIE · CCC code: 0277-786X/14/\$18 - doi: 10.1117/12.2041187 
region diffuses into interior regions of the thin film. Different crystallization regimes can be obtained according to the different process parameters.

- If the laser power used is large enough the a-Si thin film is melted, leading to liquid phase crystallization (LPC), this phenomenon is based on the facts that the melting point of a-Si is lower compared to c-Si. This phenomenon can be observed with reflectivity measurements, where once the a-Si melting temperature is reached the reflectivity drops until the c-Si is also melted (at a higher temperature) [19]. This regime is typical when using pulsed lasers, since the pulse duration times are very short and the peak powers are very high. The grain size originated in the supercooled liquid after laser melting is determined by the competition between nucleation and growth. High nucleation rate combined with slow growth rate leads to many small grains whereas low nucleation rate combined with high growth rate leads to few large grains [20].

- Above the ablation threshold the material is removed and some damage is generated in the silicon. This regime should be avoided when using CW lasers.

- Due to the metastable character of amorphous silicon, below the melting temperature of a-Si, crystallization can also be achieved by a solid phase process (SPC). Depending on crystallization temperature and on the a-Si properties (e.g. deposition conditions, interfaces, substrates,-...) nucleation and growth take place at different rates. This process is typically done using furnace annealing, but it can also be the achieved using $\mathrm{CW}$ lasers and slow scanning speeds.

In the present work, we report experimental analysis of a-Si local crystallization by $\mathrm{CW}$ green laser irradiation in the $\mathrm{ms}$ time range. The samples are irradiated with different laser powers and during different irradiation times in order to evaluate the effect of these parameters on the a-Si crystallization. Numerical simulation is used to help to understand the different physical mechanisms that take place in the crystallization process.

\section{NUMERICAL MODEL}

In order to acquire detailed knowledge of Si crystallization it is essential to understand the evolution of the temperature distribution in time and space [1]. Depending on the time duration of the phase-change process several models are used $[6,9,10,15,16]$.Although some experimentally identified mechanisms are represented well in computational modelling, even advanced models cannot describe all experimental data. In this study a simple thermal model is presented.

In this model, the material is heated by a CW laser during a time (in the order of ms) resulting in a fast increase of the aSi surface temperature. When $T_{m}(1420 \mathrm{~K})$ temperature is reached, the surface begins to melt. Then, the temperature of the melting layer will remain constant until the energy needed for phase change is absorbed. The crystallization of a-Si can take place not only via melting, but also via solid phase crystallization (SPC). Nevertheless, the SPC mechanism is not considered in our numerical model.

Depending on the laser fluence, the vaporization temperature $T_{v}$ can be reached at the centre of the irradiated area. In these cases ablation effects are expected and the annealing process becomes ineffective. In the results presented in this paper, ablation was never observed and it has not been taken into account in the model.

The incident energy of the laser pulse is partially absorbed raising the temperature of the silicon layer. When the material is melted and cools down then solidifies to poly-Si phase. The heating process in the a-Si thin film is governed by the general heat transfer equation:

$$
\rho(T) C_{p}(T) \frac{d T}{d t}=\nabla[k(T) \nabla T]+S
$$

Where $T$ is the temperature, $\rho$ is the material density, $C_{p}$ the specific heat and $k$ the thermal conductivity, when a heat source $S$ is considered.

The source term $(S)$ in Equation 1 refers to the incident laser beam in the model, which has a Gaussian profile for the irradiance distribution and a boxcar function (switch-on/switch-off $\mathrm{CW}$ laser) for the time evolution, while the depth distribution follows the Beer-Lambert law taking into account the laser wavelength $(532 \mathrm{~nm})$. So, the whole source term $S(r, z, t)$ can be described by the following equation: 


$$
S(r, z, t)=P_{i}(r, t)[(1-R(T)) \alpha(T) \exp (-\alpha(T)|z|)]
$$

Where $\alpha(T)$ is the material absorption coefficient, $R(T)$ the surface reflectivity and $P_{i}$ the incident laser power $\left(\mathrm{W} / \mathrm{m}^{2}\right)$ given by:

$$
P_{i}=\left\{\begin{array}{cc}
0 & t<0 \\
P_{p}\left(\frac{2}{\pi r_{w}^{2}}\right) \exp \left[-2\left(\frac{r}{r_{w}}\right)^{2}\right] & 0<t<\tau \\
0 & t>\tau
\end{array}\right.
$$

Where $P_{p}$ is the peak power, $r_{w}$ is the beam radius, that is, the half-width at $1 / \mathrm{e}^{2}$ of the laser intensity along the radial direction and $\tau$ is the irradiation time of the laser.

The two dimensional non-linear heat transfer equation is difficult to solve by analytical approach, so finite element method (FEM) was used to solve it numerically by means of COMSOL Multiphysics [20]. According to the mathematical model described before, a heat transfer time-dependent study is applied using Heat Transfer in Solids equations with a 2D-Axisymmetric model.

Regarding the symmetry and the boundary conditions of the problem, the model is restricted to two dimensions, $\mathrm{r}$ and $\mathrm{z}$ in cylindrical coordinates system, where $r$ is the distance to the centre of the laser pulse and $z$ the depth dimension in the material. The model sample consists of a $2 \mu \mathrm{m}$-thick a-Si layer on a glass substrate. Due to the thickness of the a-Si layer and the laser parameters used (maximum power and maximum irradiation time), the substrate is not virtually affected. The boundary conditions applied include initial room temperature, thermal insulation for right, left and bottom boundaries, and finally, surface-to-ambient radiation and convective cooling at the top surface.

The specific heat, density and thermal conductivity of the material $\left(C_{p}(T), k(T)\right)$ are assumed to be temperature dependent. The specific heat of a-Si, $C_{p}(T)$, is replaced by $\left(C_{p}(T)+\delta L_{m}\right)$ in the heat transfer equation, where $L_{m}$ is the latent heat of melting and $\delta$ is a Gaussian function given in Table I. The additional term $\delta L_{m}$ takes into account the phase change effect within the temperature range of this study [16]. Analogous treatment is made in case of liquid-vapor phase change at the corresponding temperature $T_{v}$. Absorption coefficient $\alpha(T)$ and surface reflectivity $R(T)$ are taken for 532 $\mathrm{nm}$ regarding the laser source. Thermal properties and relevant parameters used for the simulation are listed in Table 1 $[15,21,22]$.

Table 1. Thermal properties and relevant parameters used for the simulation.

\begin{tabular}{|l|l|l|}
\hline \multicolumn{1}{|c|}{ Parameters } & \multicolumn{1}{c|}{ Symbol } & \multicolumn{1}{c|}{ Value/Function } \\
\hline Beam radius $[\mu \mathrm{m}]$ & $r_{w}$ & 10 \\
\hline Absorption coefficient (solid) $\left[\mathrm{m}^{-1}\right]$ & $\alpha_{s}$ & $1.25 \mathrm{e} 7$ \\
\hline Absorption coefficient (liquid) $[\mathrm{m}-1]$ & $\alpha_{l}$ & 0.4 \\
\hline Reflectivity a-Si (solid) & $R_{s}$ & 0.73 \\
\hline Reflectivity a-Si (liquid) & $R_{l}$ & $(952+171 \cdot T / 685)$ \\
\hline Specific heat $\left[\mathrm{J} \mathrm{kg}^{-1} \mathrm{~K}^{-1}\right]$ & $C_{p}(T)$ & $\frac{1}{\sqrt{\pi} \Delta T} \exp \left(\frac{\left.T-T_{m}\right)^{2}}{\Delta T^{2}}\right)$ \\
\hline Phase change function & $\delta$ & $\left(\frac{\mathrm{e}}{2}\right)$ \\
\hline
\end{tabular}




\begin{tabular}{|l|l|l|}
\hline Thermal conductivity[W/(m·K)] & $\mathrm{k}(\mathrm{T})$ & $\begin{array}{l}(1.3 \mathrm{e}-9) \cdot(\mathrm{T}-900)^{3}+(1.3 \mathrm{e} 7) \cdot(\mathrm{T}-900)^{2}+ \\
(1 \mathrm{e}-4) \cdot(\mathrm{T}-900)+1\end{array}$ \\
\hline Latent heat (melting) $[\mathrm{J} / \mathrm{g}]$ & $L_{m}$ & 1320 \\
\hline Latent heat (vaporization) $[\mathrm{J} / \mathrm{g}]$ & $L_{v}$ & $13.6 \mathrm{e} 3$ \\
\hline Melting temperature $[\mathrm{K}]$ & $T_{m}$ & 1420 \\
\hline Boiling temperature $[\mathrm{K}]$ & $T_{v}$ & 2628 \\
\hline
\end{tabular}

The meshing of the model accounts for the laser pulse irradiation area. Therefore a finer meshing is used in the area closest to the center $(r=0)$ and a coarser meshing is used for the substrate. The simulation time is always several $\mathrm{ms}$ longer than the irradiation time with a maximum time-step of $0.01 \mathrm{~ms}$. PARDISO direct solver algorithm is used to solve the linear system [20].

As discussed later, the numerical simulation gives the temperature evolution in the sample, predicts the melt-depth and helps to understand the different crystallization mechanisms that take place in this laser process (Figure 1).
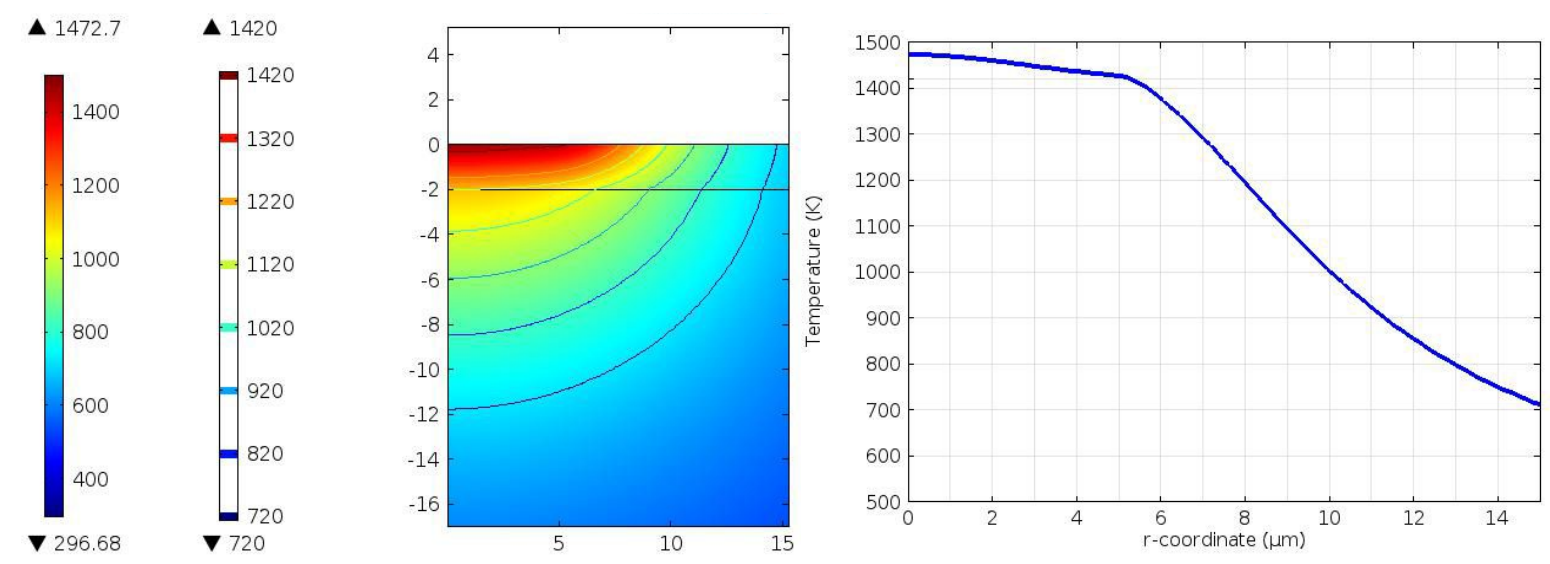

Figure 1. Maximum temperature profiles obtained with a CW laser $(532 \mathrm{~nm})$ with $124 \mathrm{~mW}$ and $10 \mathrm{~ms}$ :

(left) Depth profile (right) Surface profile. Temperaure is expressed in Kelvins (K), and the radial and depth dimensions are in microns $(\mu \mathrm{m})$.

\section{EXPERIMENTAL PROCEDURE}

Amorphous silicon films were deposited at $330^{\circ} \mathrm{C}$ onto $10 \times 10 \mathrm{~cm}^{2}$ Al-coated glass by means of a conventional PECVD reactor at $13.56 \mathrm{MHz}$ from pure silane (SiH4). Plasma conditions were fixed to a gas pressure of $400 \mathrm{mTorr}$, an RF power of $1 \mathrm{~W}$ and a $\mathrm{SiH}_{4}$ flow of $20 \mathrm{sccm}$. Deposition time was set to 6 hours to achieve a-Si:H layers with a thickness of 2 microns.

Laser irradiation was performed using a standard DPSS CW laser source (Millennia Prime, Newport-Spectra Physics) emitting at $532 \mathrm{~nm}$ with Gaussian irradiation profile. An acusto-optic modulator and an optical attenuator were placed in the optical path of the laser beam, in order to control respectively the irradiation time $(\tau)$ and the laser power $(P)$.

These two parameters were varied from 0.5 to $15 \mathrm{~ms}$ in the case of $\tau$ and from 84 to $160 \mathrm{~mW}$ in the case of $P$. That parametric window was selected to have enough energy to crystallize the a-Si samples without any morphological damage. The laser beam was focused onto the sample, leading to a spot diameter of $20 \mu \mathrm{m}$. 
Confocal laser scanning microscopy (Leica DCM3D) was used to characterize the morphology of the irradiated area, while micro-Raman spectroscopy (Renishaw, inVia Raman microscope) was used to study the structural modifications (crystallization) induced in the irradiated surface.

The Raman measurements were performed in the range from $200 \mathrm{~cm}^{-1}$ to $850 \mathrm{~cm}^{-1}$ with a $100 \mathrm{X}$ optical microscope objective, scanning the irradiation area with an incident measurement spot of $1 \mu \mathrm{m}$ diameter. The excitation optical source consisted of an $\mathrm{Ar}^{+}$laser tuned at the wavelength of $514 \mathrm{~nm}$.

Non-Irradiated samples show the usual Raman spectrum from the amorphous silicon with a broad band centred at 480 $\mathrm{cm}^{-1}$ corresponding to the TO phonon mode [24]. However, the irradiated areas show Raman spectra with additional contributions due to crystallized silicon. In order to analyse the crystalline fraction of the irradiated area, the Raman spectra were fitted by three Gaussian line profiles: a Gaussian profile contribution of amorphous silicon set at $480 \mathrm{~cm}^{-1}$ and two other Gaussian bands are related to the contribution of the nanocrystalline silicon (Gaussian peak centered on $514 \mathrm{~cm}^{-1}$ ) and surface modes or silicon defects. This method yields only a semi-quantitative estimation of the crystalline fraction of the irradiated sample, although it is good enough for describing the dependence of the crystallization with the laser power and the irradiation time $[25,26]$.

\section{RESULTS AND DISCUSSION}

The experimental setup has been design to allow an accurate control over the irradiation time $(\tau)$ and the laser power $(P)$. When the annealing process is done with $\mathrm{CW}$ lasers (with powers in the range of $100 \mathrm{~mW}$ and irradiation times in the order of several milliseconds) the irradiation time must be a critical parameter, since it is long enough to allow solid phase crystallization in addition to liquid phase crystallization. The laser power is also a critical parameter as it has a direct effect on the maximum temperature achieved and therefore on increasing the crystal growth rate.

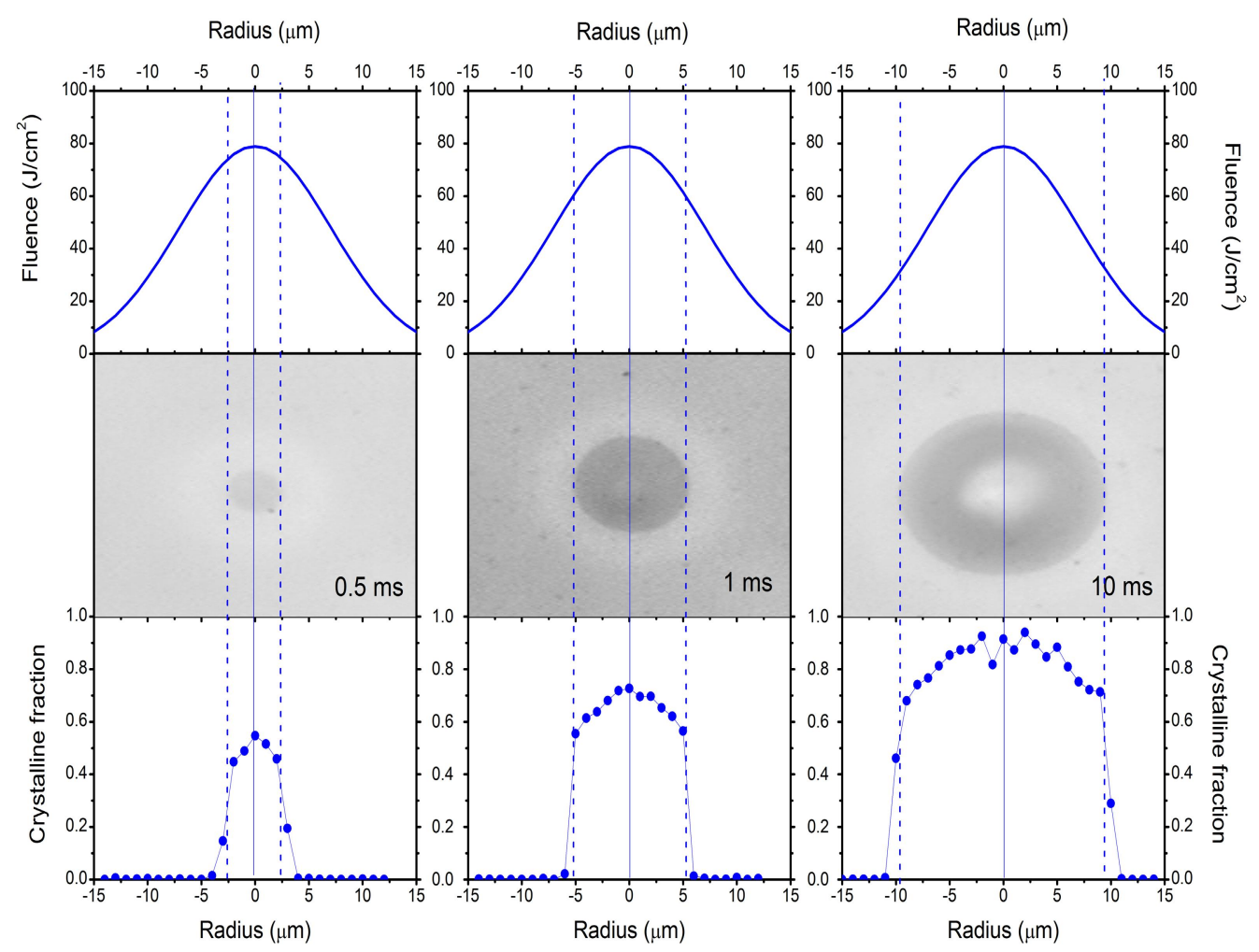

Figure 2. (Middle) Images of crystallized spots using $124 \mathrm{~mW}$ and three different irradiation times: (left) $0.5 \mathrm{~ms}$ (centre) 1.0 $\mathrm{ms}$ and (right) $10.0 \mathrm{~ms}$ and (below) the measured crystalline fraction in function of the distance to the centre of the spot. The theoretical Gaussian distribution of fluencies in the laser beam is also shown (top). Dashed lines indicate the visual diameter of the crystallized spot. 
Figure 2 shows images of the irradiated spot when using three different $\tau$ values $(0.5,1$, and $10 \mathrm{~ms})$ while $\mathrm{P}$ is fixed to a value of $124 \mathrm{~mW}$. The crystallized area can be easily identified as the darkened area. The Figure shows also the theoretical fluence distribution onto the sample for a Gaussian laser beam with this $P$ value, which is logically the same for the three $\tau$ values. However, the spot size clearly increases as $\tau$ increases. Spots have circular symmetry due to the Gaussian distribution of the laser energy, and therefore the crystalline fraction has been measured at different distances from the centre of the spot (Figure 2). Crystalline fraction values larger than 0.4 fit to the darkened area of the spot. The crystalline fraction is also larger in the centre of the spots and decreases as the radius increases, due to the Gaussian nature of the laser beam.

Figure 3 shows the crystalline fraction as a function of the distance to the centre of the laser spot for different irradiation times and a fixed laser power of $124 \mathrm{~mW}$. The crystalline spot size clearly increases as $\tau$ increases, although the enhancement is not linear and for longer irradiation times $(5-10 \mathrm{~ms})$ the size is very similar. The maximum value of the crystalline fraction, which is located in the center of each spot, increases as $\tau$ increases, from 0.55 at $1 \mathrm{~ms}$ to around 0.85 at $10 \mathrm{~ms}$. These differences are related with the fact that Raman excitation laser has an optical penetration depth of several hundreds of nanometers and then structural information is collected from a whole volume of the film. As $\tau$ increases, the energy transferred to the film increases and also a larger volume of the film is heated due to heat transfer. Therefore the crystalline fraction should increase.

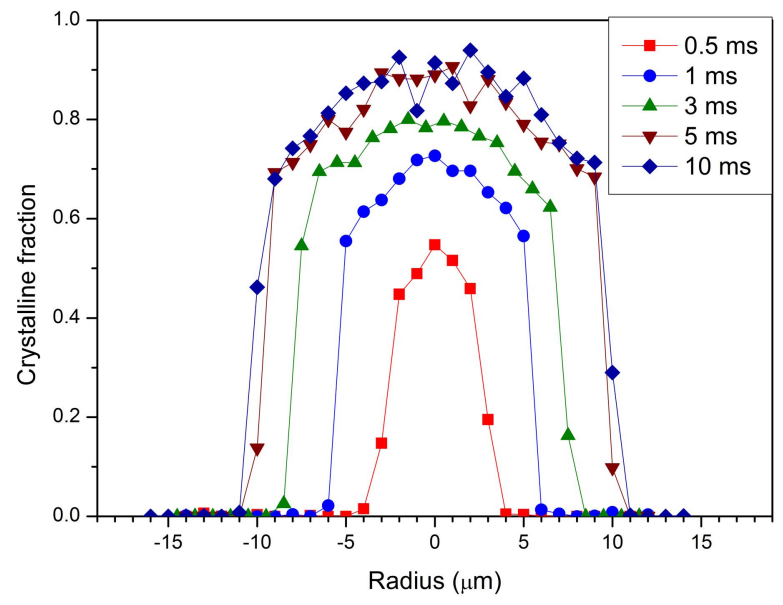

Figure 3. Crystalline fraction as a function of the distance to the centre of the laser spot for different irradiation times and a fixed laser power of $124 \mathrm{~mW}$. The lines are drawn to help visualization.

These experimental results can be then correlated to the temperature reached by the sample, calculated using the model described above. Figure 4 shows the simulated maximum temperature reached on the surface of the sample as a function of the radius for the same three $\tau$ values shown in Figure 2. When comparing these temperatures with the crystalline fraction, it is shown at the shortest $\tau$ that the crystallized area reaches a surface temperature of at least $1430 \mathrm{~K}$. This value corresponds to the melting temperature of a-Si $(1420 \mathrm{~K}$ in Table 1) so film crystallization has occurred through LPC.

However, at longer irradiation times there is also crystallization in areas with maximum surface temperature below 1420 $\mathrm{K}$. This effect can be seen more clearly when studying how the temperature evolves inside the film (Figure 5). The film volume in which the temperature is higher than $1420 \mathrm{~K}$ is quite similar for all the irradiation times (in the order of the $\mathrm{ms}$ ), although at long irradiation times the crystalline fraction yielded is much larger in value, i.e. more volume of a-Si is crystallized. If the minimum temperature needed to crystallize is established from comparing the experimental crystallized area and the simulated data $(1360 \mathrm{~K}$ for $1 \mathrm{~ms}$ and $910 \mathrm{~K}$ for $10 \mathrm{~ms}$ in Figure 4), it is possible to calculate the volume heated up to these or higher temperatures. These volumes are much larger at longer $\tau$ values and can justify the differences shown in the crystalline fraction. 
From these figures one can deduce that for the longer times all thickness of the a-Si substrate has been crystallized, while for shorter pulses a combination of effects are present and for even shorter pulses crystallization only takes place in the melted volume.

Then, our hypothesis is that the film crystallization is a combination of two mechanisms:

- In the melted area (the central area of the spot) liquid phase crystallization is taking place.

- In the outermost regions and the deeper areas, the temperature is not sufficient to produce LPC, and crystallization can only take place due to solid state crystallization or via solid state epitaxy due to the presence of crystallized Si in the centre of the spot via LPC.

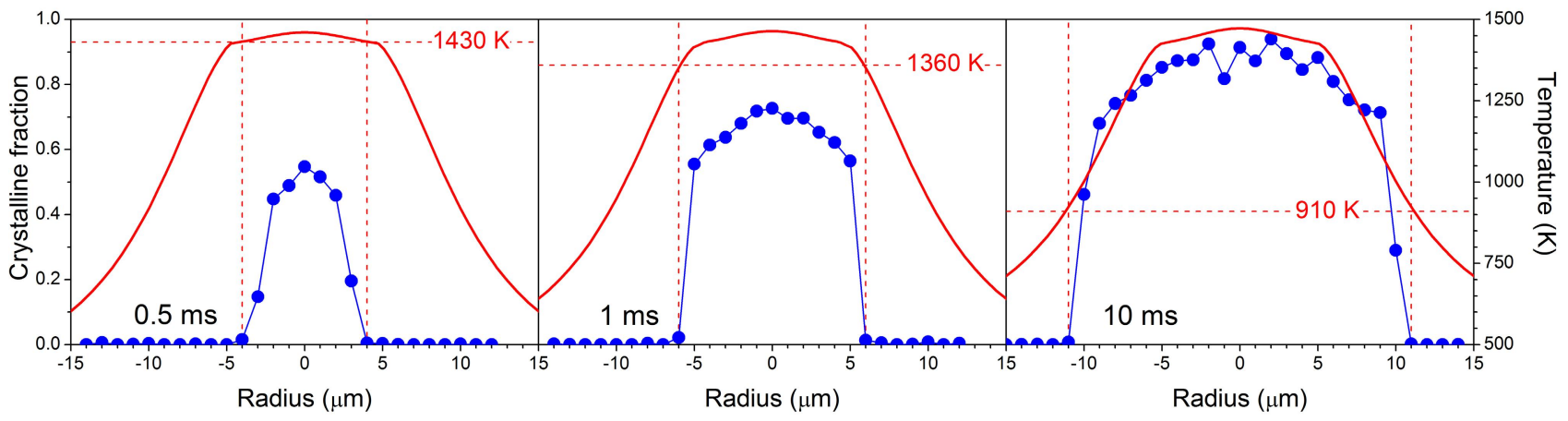

Figure 4. (Red solid lines, right axis) Simulated maximum temperature reached on the surface of the sample at the end of the laser irradiation and (blue circles, left axis) experimental crystalline fraction as a function of the radius for different irradiation times and a fixed power of $124 \mathrm{~mW}$ : (left) $0.5 \mathrm{~ms}$; (centre) $1 \mathrm{~ms}$; (right) $10 \mathrm{~ms}$. Red dashed lines indicated the diameter of the spot in which the measured crystalline fraction is non negligible and the corresponding simulated temperature.

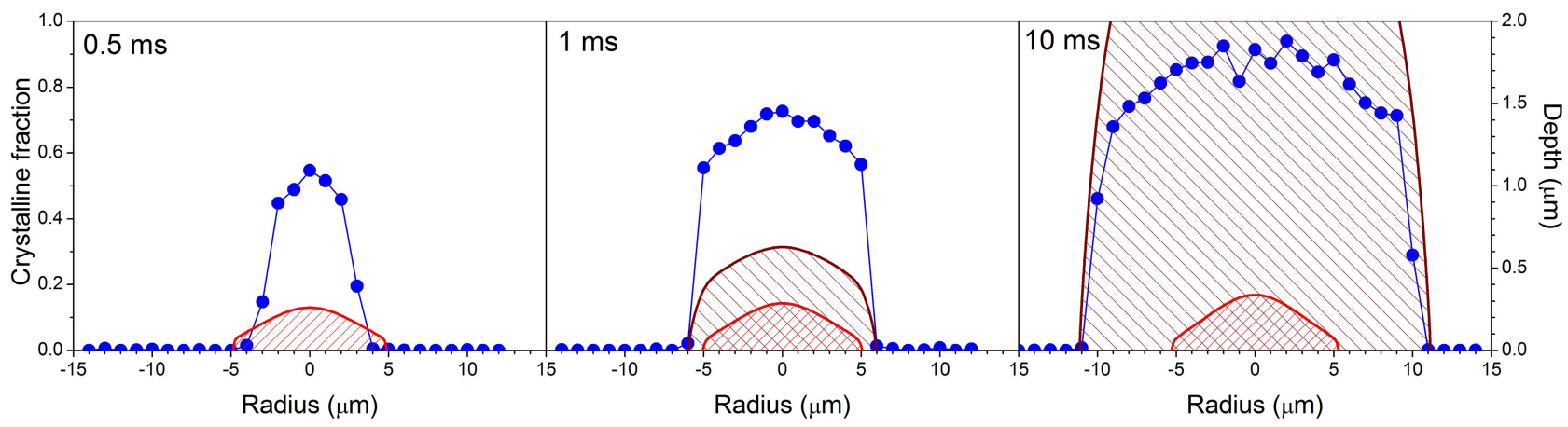

Figure 5. Depth into the sample in which the simulated temperature has reached at least $1420 \mathrm{~K}$ (light red filled areas) as a function of the radius for different irradiation times and a fixed power of $124 \mathrm{~mW}$ : (left) $0.5 \mathrm{~ms}$; (centre) $1 \mathrm{~ms}$; (right) $10 \mathrm{~ms}$. In the case of 1 and $10 \mathrm{~ms}$ it is also shown the regions in which at least temperatures of (dark red filled areas) 1360 and 910 $\mathrm{K}$ respectively are also reached. Those temperature values are experimental determined from data in Figure 4.

The effect of laser power has been studied in a large range of power values in the centre of the laser spot (Figure 6), since the crystalline fraction in that point mirrors the volume of crystallized a-Si in the sample according to the previous results. It is important to notice that in the centre of the laser spot LPC will always occur except for very low $P$ or $\tau$ values.

Figure 6 shows the crystalline fraction measured in the centre of the irradiated spot as a function of laser power for different irradiation times. The crystalline fraction shows similar behavior regardless of $\tau$ when increasing $P$. For low powers the sample remains amorphous up to a certain threshold value, in which the crystalline fraction suddenly 
increases up to values larger than 0.5 . This $P$ value can be identified as the laser power needed to start LPC. From that on, the crystalline fraction seems to scale linearly with $P$. Moreover, when crystallization starts the value obtained for longer irradiation times is larger, that could be related to SPC because the sample has remained at a high temperature but below melting temperature during more time, increasing the contribution of SPC to film crystallization

The power threshold, in which the sample starts to crystallize, depends on the irradiation time, since as shorter is $\tau$, lower is the value of P needed to start the crystallization. This can be understood in terms of the energy that is necessary to put into the sample increasing its temperature high enough to crystallize: as shorter is $\tau$, larger values of $P$ are needed to led to the same temperature increment. However, this effect is not linear because large $\tau$ values show similar values of power threshold and there should be another temporal factors implied during crystallization. This can also be observed if the temperature evolution is studied from the simulations, although the maximum temperature is very similar, the temporal profile difers, what could explain many of the observed effects. As it can be seen from these experiments the crystallization process is a very complex process were not only the temperature but also the temperature history, the temperature gradient and the existence of a-Si/c-Si interfaces can have a great effect on the crystalline fraction.

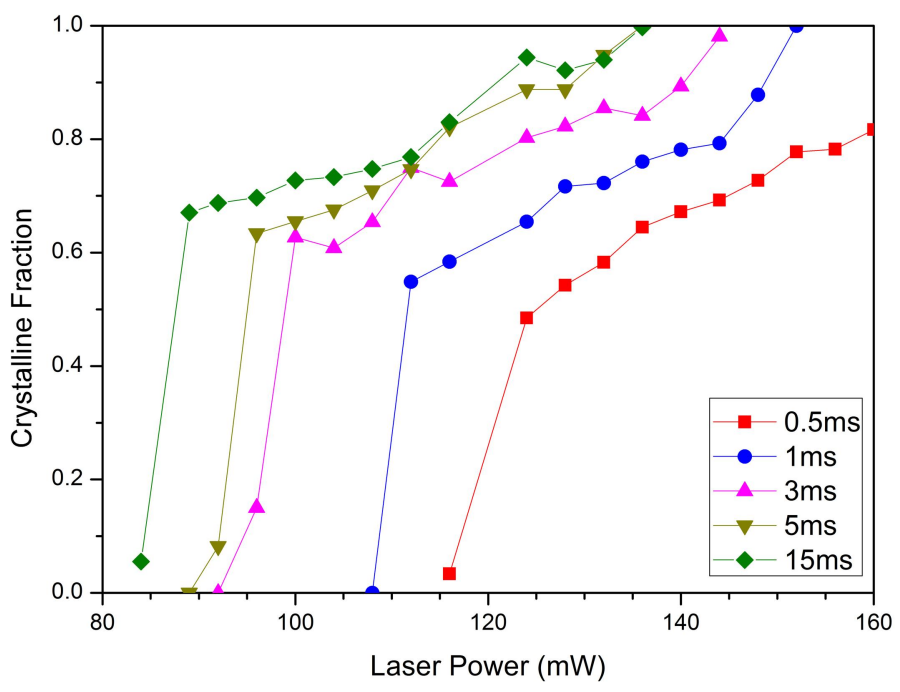

Figure 6. Crystalline fraction in the centre of the crystallized spot as a function of the laser power for different irradiation times.

\section{CONCLUSIONS}

$\mathrm{CW}$ green laser crystallization of a-Si:H films (with a thickness of $2 \mu \mathrm{m}$ ) deposited by PECVD has been experimentally investigated.

Laser irradiated spots were produced by using different laser powers and irradiation times. Experimental values from Raman spectra measurements were used to determine the local crystalline fraction. Process dependence on power and irradiation time has been studied. These parameters are identified as key variables in the crystallization process. The power threshold for starting the crystallization is reduced as the irradiation time is increased. Once this threshold is achieved, crystalline fraction increases lineally with power for each irradiation time.

A transient heat conduction numerical model was implemented to simulate the process and the experimental results are analysed with the aid of this model. The presence of two crystallization mechanisms, liquid phase crystallization (due to melting) and solid phase crystallization is deduced from the maximum temperature distribution in the sample (obtained in the simulations).

The possibility of using CW green laser to obtain poly-Si from a-Si:H films has been demonstrated. The use of new $\mathrm{CW}$ green laser sources could open the viability of using this process for industrial photovoltaic applications. 


\section{ACKNOWLEDGEMENTS}

This work has been supported by the Spanish Ministry of Science and Innovation under projects AMIC ENE201021384-C04-02, SIMLASPV ENE2011-23359 and INNDISOL IPT-420000-2010-6 (FEDER funded "Una manera de hacer Europa").

\section{REFERENCES}

[1] Nickel, N. H., [Laser Crystallization of Silicon - Fundamentals to Devices, Semiconductors \& Semimetals, Volume 75], Elsevier Academic Press, Amsterdam (2003).

[2] Brotherton, S.D., "Polycrystalline silicon thin film transistors," Semicond. Sci. Technol. 10,721-738 (1995).

[3] Shah, A.V., Schade, H., Vanecek, M., Meier, J., Vallat-Sauvain, E., Wyrsch, N. , Kroll, U., Droz, C., Bailat, J., "Thin-film silicon solar cell technology," Prog Photovolt.: Res. Appl., 12, 113-142 (2004).

[4] Ecoffey, S., Bouvet, D., Ionescu, A.M., Fazan, P., "Low-pressure chemical vapor deposition of nanograin polysilicon ultra-thin films," Nanotechnology 13, 290-293 (2002).

[5] Voutsas, A.T., "A new era of crystallization: advances in polysilicon crystallization and crystal engineering," Appl. Surf. Sci. 208, 250-262 (2003).

[6] Chen, Y-R., Chang, C-H., Chao, L-S., "Modeling and experimental analysis in excimer-laser crystallization of a-Si films," Journal of Crystal Growth 303, 199-202 (2007).

[7] Azuma, H., Takeuchi, A., Ito, T., Fukushima, H., Motohiro, T., Yamaguchi, M., "Pulsed KrF excimer laser annealing of silicon solar cell," Solar Energy Materials \& Solar Cells 74, 289-294 (2002).

[8] Sameshima. T., "Laser crystallization for large-area electronics," Appl. Phys. A 96 (1), 137-144 (2009).

[9] Palani, I.A., Vasa, N.J., Singaperumal, M., Okada, T., "Investigation on Laser-annealing and Subsequent Lasernanotexturing of Amorphous Silicon (a-Si) Films for Photovoltaic Application," JLMN-Journal of Laser Micro/Nanoengineering 5 (2), 150-155 (2010).

[10] García, O., García-Ballesteros, J. J., Munoz-Martin, D., Núñez-Sánchez, S., Morales, M., Carabe, J., Torres, I., Gandía, J.J., Molpeceres, C., "Estimation of Local Crystallization of a-Si:H Thin Films by Nanosecond Pulsed Laser Irradiation Through Local Temperature Simulation," Physics Procedia 39, 286-294 (2012).

[11] Hara, A., Takeuchi, F., Takei, M., "High-performance polycrystal- line silicon thin film transistors on nonalkali glass produced using continuous wave laser lateral crystallization,” Jpn. J. Appl. Phys. 41, L311-L313 (2002).

[12] Dassow, R., Kohler, J.R., Helen, Y., Mourgues, K., Bonnaud, O., Mohammed-Brahim, T., Werner, J.H., "Laser crystallization of silicon for high-performance thin-film transistors," Semicond. Sci. Technol. 15, L31-L34 (2000).

[13] Park, S., Ku, Y., Kim, E., Jang, J., Kim, K., Kim, C., "Selective crystallization of amorphous silicon thin film by a CW green laser," J. Non-Cryst. Solids 352, 993-997 (2006).

[14] Saboundji, A., Mohammed-Brahim, T., Andrä, G., Bergmann, J., Falk, F., "Thin film transistors on large single crystalline regions of silicon induced by CW laser crystallization," J. Non-Cryst. Solids 338-340, 758-761 (2004).

[15] Yuan, Z., Lou, Q., Zhou, J., Dong, J., Wei, J., Wang, Z., Zhao, H., Wu, G., "Numerical and experimental analysis on green laser crystallization of amorphous silicon thin films," Optics \& Laser Technology 41, 380383 (2009).

[16] Said-Bacar, Z., Leroy, Y., Antoni, F., Slaoui, A., Fogarassy, E., "Modeling of CW laser diode irradiation of amorphous silicon films," Appl. Surf. Sci. 257, 5127-5131 (2011).

[17] Dore, J., Evans, R., Schubert, U., Eggleston, B. D., Ong, D., Kim, K., Huang, J., Kunz, O., Keevers, M., Egan, R., Varlamov, S., Green, M. A., "Thin-film polycrystalline silicon solar cells formed by diode laser crystallization,” Prog. Photovolt: Res. Appl. 21, 1377-1383 (2013).

[18] Michaud, J.F., Rogel, R., Mohammed-Brahim, T., Sarret, M., "CW argon laser crystallization of silicon films: Structural properties," Journal of Non-Crystalline Solids 352, 998-1002 (2006). 
[19] Schmidt, T., Gawlik, A., Schneidewind, H., Ihring, A., Andrä, G., Falk, F., "Experimental setup for investigating silicon solid phase crystallization at high temperatures," Optics Express 21(14), 16296 - 16304 (2013).

[20]Falk, F., Andrä, G., "Laser crystallization - a way to produce crystalline silicon films on glass or on polymer substrates," Journal of Crystal Growth 287, 397-401 (2006).

[21] COMSOL Multiphysics Reference Manual, version 4.3, COMSOL AB. (2013).

[22] Ong, C.K., Tan, H.S., Sin, E.H., "Calculations of Melting Threshold Energies of Crystalline and Amorphous Materials Due to Pulsed-laser Irradiation," Materials Science and Engineering 79, 79-85 (1986).

[23] Poulain, G., Blanc, D., Kaminski, A., Semmache, B., Lemiti, M., "Modeling of Laser Processing for Advanced Silicon Solar Cells," Excerpt from the Proceedings of the COMSOL Conference 2010 Paris

[24] Smit, C., van Swaaij, R.A.C.M., Donker, H., Petit, A.M.H.N., Kessels, W.M.M., van de Sanden, M.C.M., "Determining the material structure of microcrystalline silicon from Raman spectra," Journal of Applied Physics 94 (5), 3582-3588 (2003).

[25] Houben, L., Luysberg, M., Hapke, P., Carius, R., Finger, F., Wagner, H., "Structural properties of microcrystalline silicon in the transition from highly crystalline to amorphous growth," Philosophical Magazine A 77(6), 1447-1460 (1998).

[26] Iqbal, Z., Veprek, S., Webb, A.P., Capezzuto, P., "Raman-scattering from small particle-size polycrystalline silicon," Solid State Communications 37 (12), 993-996 (1981). 